\title{
Influence of systemic fluoroquinolone administration on the presence of Pasteurella multocida in the upper respiratory tract of clinically healthy calves Boudewijn Catry*1,4,5, Siska Croubels ${ }^{3}$, Stefan Schwarz ${ }^{6}$, Piet Deprez ${ }^{2}$, Bianca Cox ${ }^{5}$, Corinna Kehrenberg ${ }^{6}$, Geert Opsomer ${ }^{1}$, Annemie Decostere ${ }^{4}$ and Freddy Haesebrouck ${ }^{4}$
}

\author{
Address: ${ }^{1}$ Department of Reproduction, Obstetrics and Herd Health, Faculty of Veterinary Medicine, Salisburylaan, 133, Ghent University, 9820 \\ Merelbeke, Belgium, 2Department of Internal Medicine and Clinical Biology of Large Animals, Faculty of Veterinary Medicine, Salisburylaan, 133, \\ Ghent University, 9820 Merelbeke, Belgium, ${ }^{3}$ Department of Pharmacology, Toxicology and Biochemistry, Faculty of Veterinary Medicine, \\ Salisburylaan, 133, Ghent University, 9820 Merelbeke, Belgium, ${ }^{4}$ Department of Pathology, Bacteriology, and Poultry Diseases, Faculty of \\ Veterinary Medicine, Salisburylaan, 133, Ghent University, 9820 Merelbeke, Belgium, ${ }^{5}$ Scientific Institute of Public Health, Rue Juliette \\ Wytsmanstraat 14, 1050 Brussels, Belgium and ' Institute of Farm Animal Genetics, Friedrich-Loeffler-Institute (FLI), Hoeltystr. 10,31535 \\ Neustadt-Mariensee, Germany \\ Email: Boudewijn Catry* - Boudewijn.Catry@iph.fgov.be; Siska Croubels - Siska.croubels@ugent.be; \\ Stefan Schwarz - stefan.schwarz@fli.bund.de; Piet Deprez - Piet.deprez@ugent.be; Bianca Cox - Bianca.Cox@iph.fgov.be; \\ Corinna Kehrenberg - corinna.kehrenberg@fli.bund.de; Geert Opsomer - Geert.Opsomer@ugent.be; \\ Annemie Decostere - Annemie.Decostere@ugent.be; Freddy Haesebrouck - freddy.haesebrouck@ugent.be \\ * Corresponding author
}

Published: 22 September 2008

Acta Veterinaria Scandinavica 2008, 50:36 doi:10.1/86/I75I-0I47-50-36
Received: 5 March 2008

Accepted: 22 September 2008

This article is available from: http://www.actavetscand.com/content/50/I/36

(C) 2008 Catry et al; licensee BioMed Central Ltd.

This is an Open Access article distributed under the terms of the Creative Commons Attribution License (http://creativecommons.org/licenses/by/2.0), which permits unrestricted use, distribution, and reproduction in any medium, provided the original work is properly cited.

\begin{abstract}
The influence of enrofloxacin administration $(5 \mathrm{mg} / \mathrm{kg})$ for five consecutive days on the occurrence of Pasteurella multocida in the upper respiratory tract of two healthy calves was monitored over a 10-day period. From nasal swabs of two additional healthy control calves, which received a placebo saline administration, $P$. multocida was isolated throughout the study period. In the enrofloxacin treated calves, $P$. multocida was not demonstrated in the nasopharynx from $48 \mathrm{~h}$ after the first injection until two days after the last administration, when $P$. multocida reappeared and proved to be clonal in nature to the original isolates. During the experiment, no change in minimal inhibitory concentration for enrofloxacin of the $P$. multocida isolates was detected (MIC $\leq 0.015 \mu \mathrm{g} / \mathrm{mL}$ ). Enrofloxacin concentrations were determined in the plasma by a high-performance liquid chromatography method with fluorescence detection. The PK/PD indices AUC/MIC and $C_{\max } / \mathrm{MIC}$ ratio were calculated and found to be II 57.7 and 129.8 , respectively. Remarkably, the respiratory pathogen Arcanobacterium pyogenes became the predominant recovered organism in the nasopharynx of one animal following enrofloxacin therapy throughout the remaining of the experiment.
\end{abstract}

\section{Findings}

In calves, enrofloxacin is frequently used to treat pneumonic pasteurellosis, a disease mostly due to Pasteurella multocida [1]. P. multocida is a common inhabitant of the upper respiratory tract of calves. To better understand the epidemiology of pneumonic pasteurellosis and the occur- 
rence of antimicrobial resistance, knowledge is needed on how systemic fluoroquinolone administration affects the flora of the nasopharynx in healthy calves. This is important as metaphylaxis is a common practice in the prevention of bovine respiratory diseases. Preventive treatment of "at risk animals" may be associated with a selection pressure leading to antimicrobial resistance or a shift in the population of bacteria present in the nasopharynx.

The aim of the present experiment was to evaluate the influence of consecutive systemic enrofloxacin administrations on the presence and susceptibilities of $P$. multocida strains naturally present in the nasopharynx of clinically healthy calves and to find a relationship with pharmacokinetic/pharmacodynamic surrogate parameters.

Four dairy calves aged 24-28 days were loose grouphoused together during a 5-day pre-experimental period after which the calves were randomly assigned to either a treatment group or a control group. Inclusion criteria were the absence of disease and antimicrobial therapy since birth. The calves were housed two by two in straw-bedded pens (approximately $18 \mathrm{~m}^{2}$ of floor space per group) in the same automated ventilated stable $\left(17 \pm 2{ }^{\circ} \mathrm{C}\right)$, but divided by full wooden partitions approximately $1.4 \mathrm{~m}$ high. Management and hygienic measures were set up to prevent direct contact between the two study groups. Water and hay were supplied ad libitum, and the calves were maintained on an antibiotic-free milk replacer diet twice a day. Clinical observations were carried out and all calves remained healthy during the entire experiment.

The study lasted for 10 days (D0-D9). For five consecutive days (D0-D4), the two calves of the treatment group (weights at D0: 47.0 and $49.2 \mathrm{~kg}$ ) were injected intramuscularly with $5 \mathrm{mg} / \mathrm{kg}$ enrofloxacin (Baytril 2.5\%, Bayer, Milan, Italy), while the two calves of the control group (weights at Do: 48.7 and $50.0 \mathrm{~kg}$ ) received a placebo (5 $\mathrm{mL}$ isotonic saline intramuscularly). After disinfection of the nostrils with $90 \%$ ethanol, nasal samples were collected using cotton swabs inserted $10-15 \mathrm{~cm}$ into the dorsal conchae (Venturi Transsystem, Copan, Italy) every 12 h starting from D0 until D5 and on D6 and D9. Samples were cooled $\left(<7^{\circ} \mathrm{C}\right)$ and further processed within $24 \mathrm{~h}$, starting by vortexing each swab in $3 \mathrm{~mL}$ phosphate buffered saline for $10 \mathrm{~s}$. Plasma samples obtained by centrifugation of blood at $4 \times \mathrm{g}$ for $10 \mathrm{~min}$ were taken on D0 at 0 , $2,4,6,12,24 \mathrm{~h}$, on D1 at $48 \mathrm{~h}$, on D2 at $72 \mathrm{~h}$, on D3 at $96 \mathrm{~h}$, and on D4 at 100, 104, 108, 112, 120, 144 and 216 $\mathrm{h}$ (the latter intensity to explore the elimination phase) from the treated calves and from the placebo calves at $0 \mathrm{~h}$ (D0), and stored at $-20^{\circ} \mathrm{C}$ prior to assay. The experimental protocol was approved by the local ethics committee.
In the nasal samples, the numbers of enrofloxacin resistant $P$. multocida isolates and the total numbers of $P$. multocida isolates were determined using a comparative enumerating procedure (duplicate aliquots of $25 \mu \mathrm{L}$ ) on Columbia agar (Oxoid, Hampshire, UK) to which sheep blood (5\% vol/vol) and $16 \mu \mathrm{g} / \mathrm{mL}$ bacitracin ( $1 \mu \mathrm{g}$ equals 0.0654 U, Sigma Poole, UK) was added with the following concentrations of enrofloxacin (Baytril 2.5\%): 0; 0.06; $0.125 ; 0.25 ; 0.5$, and $1 \mu \mathrm{g} / \mathrm{mL}$. Reading was performed after $24 \mathrm{~h}$ and $48 \mathrm{~h}$ of aerobic incubation at $37^{\circ} \mathrm{C}$, and consisted of counting colony-forming units (CFU) distributed over each drop zone and averaged for duplicates. The species identification of one $P$. multocida colony per animal per day was confirmed by means of phenotyping and tDNA-PCR and clonality was examined by means of pulsed-field gel electrophoresis (PFGE) [1]. Bacteriological counts were expressed as median and interquartile range, and a non-parametric multivariate analysis of variance (nonparametric MANOVA) for repeated measurements and small sample sizes [2] was performed (SAS version 9.1, Sat Institute Inc., Cary, NC). Evolution of $P$. multocida recovery from the nasal swabs on media without enrofloxacin in both the treated calves and the control calves is given in Figure 1. The non-parametric MANOVA showed that the difference in $P$. multocida isolation was significant between the treatment and the placebo group over time (treatment* time $P=0.04$, time $P=0.03$ ). $P$. multocida was not recovered during the entire experiment on media containing any of the enrofloxacin concentrations. Additional susceptibility testing [1] (range 0.015-1 $\mu \mathrm{g} / \mathrm{mL}$ ) confirmed no detectable increase in enrofloxacin MIC $(\leq 0.015 \mu \mathrm{g} / \mathrm{mL})$ of seven $P$. multocida isolates recovered from both treated calves on D0 (2) and on D9 (2) and from the control calves on D0 (1) and D9 (2), and PFGE fingerprinting patterns were identical. Whether the clonally identical organisms reappeared in the treated calves either through airborne transmission from the control calves or endogenously via undetected strains, e.g. in the tonsils, is unclear.

To evaluate whether the microbiological effects of fluoroquinolone administration were in line with the current understanding of pharmacokinetic/pharmacodynamic (PK/PD) relationships, two PK/PD parameters were calculated: the maximum plasma concentration/minimal inhibitory concentration $\left(\mathrm{C}_{\max } / \mathrm{MIC}\right)$ ratio and the area under the inhibitory curve (AUC/MIC). Theoretically, $\mathrm{C}_{\max } / \mathrm{MIC}$ should exceed 10 and AUC/MIC (AUIC) should exceed 125 to minimize the selection for resistant organisms by bacterial killing also of less susceptible subpopulations (eradication) $[3,4]$.

Plasma concentrations of enrofloxacin and its active metabolite ciprofloxacin were determined using a validated high-performance liquid chromatography method 


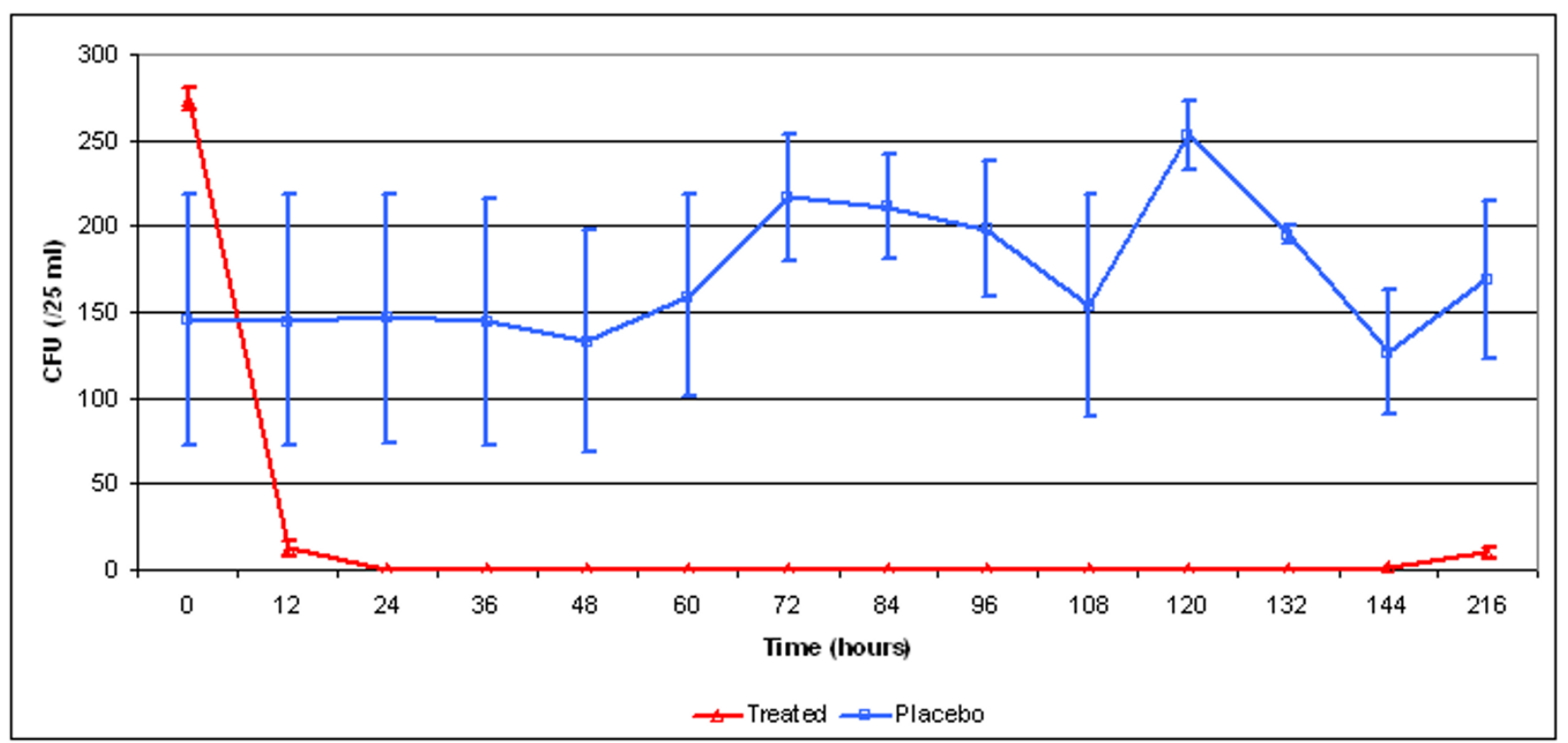

Figure I

Recovery of Pasteurella multocida (colony forming units, CFU) in the nasopharynx of calves treated with enrofloxacin and control calves on media without enrofloxacin. Error bars indicate median and interquartile range.

(HPLC) with fluorescence detection. Extraction was performed as described by Manceau et al. [5], with minor modifications. Pharmacokinetic analysis was performed using MW/Pharm software (version 3.60, Medi Ware, Utrecht, The Netherlands). The plasma concentrationtime profile could be adequately fitted to a one compartmental model $\left(\mathrm{r}^{2} \geq 0.996\right.$ for enrofloxacin and $\mathrm{r}^{2} \geq 0.973$ for ciprofloxacin). All concentrations in the placebo calves were below the limit of detection $(4.6 \mathrm{ng} / \mathrm{mL})$. Maximal plasma concentration $\left(\mathrm{C}_{\max }\right)$, elimination rate constant $\left(k_{e}\right)$ and elimination half-life $\left(\mathrm{T}_{1 / 2 \mathrm{e}}\right)$ were derived from the model. The area under the curve from time zero to infinity after the first dose $\left(\mathrm{AUC}_{0 \rightarrow \infty}\right)$ was calculated using the linear trapezoidal method for $\mathrm{AUC}_{0 \rightarrow \mathrm{t}}$ and adding the estimated terminal portion of the curve $\left(\mathrm{Ct} / \mathrm{k}_{\mathrm{e}}\right)$, where $\mathrm{t}$ is the last time of measurable plasma concentrations after the first dose. Enrofloxacin is de-ethylated into ciprofloxacin, but the degree of this metabolic process substantially varies within animal species. The mean ratio in $\mathrm{AUC}_{0 \rightarrow \infty}$ of ciprofloxacin/enrofloxacin after the first dose found here was $12.3 \%$. This was significantly lower than reported in 8-month-old buffalo calves (27\%) and adult cattle (29.9\%) [6]. In newborn and one-week-old calves the ciprofloxacin/enrofloxacin ratio can range from 10 to $27 \%$. The ratio is probably lower in young calves due to the lower metabolic capacity at this age [7]. The area under the concentration-time curve at steady-state over 24 $\mathrm{h}\left(\mathrm{AUC}_{0 \rightarrow 24 \mathrm{~h}}\right)$ was set equivalent to the $\mathrm{AUC}_{0 \rightarrow \infty}$ after the first dose. The ratio of $\mathrm{AUC}_{0 \rightarrow 24} \mathrm{~h} / \mathrm{MIC}$ (AUIC) and plasma $\mathrm{C}_{\max } / \mathrm{MIC}$ was expressed as a dimensionless value. For the isolated P. multocida strains the mean AUIC and $\mathrm{C}_{\max } / \mathrm{MIC}$ for enrofloxacin were found to be 1157.7 and 129.8, respectively (MIC for enrofloxacin $\leq 0.015 \mu \mathrm{g} / \mathrm{mL}$ ). Even when a conservative MIC of $0.06 \mu \mathrm{g} / \mathrm{mL}$ [1] is taken into account, the thresholds would successfully be exceeded (289.4 for AUIC; 32.4 for $\mathrm{C}_{\max } / \mathrm{MIC}$ ). Unfortunately, the obtained values rely on the plasma concentrations and not on concentrations measured in the nasopharynx. Nevertheless, several studies dealing with pharmacokinetics of fluoroquinolones in both plasma and at the site of infection are available in cattle and in line with our observations $[8,9]$. Recently, it has been shown that fluoroquinolones are a substrate for ATP-dependent efflux transporters which may result in effective drug concentrations in luminal compartments of target tissues $[10,11]$ In addition, during natural courses of bovine respiratory disease, the PK/PD surrogate markers for fluoroquinolones can largely exceed those seen in apparently healthy animals [12].

In one calf of the enrofloxacin treated group, a quasi pure culture of Arcanobacterium pyogenes was recovered from D2 
(2.9 $\log 10 \mathrm{CFU} / \mathrm{mL}$ ) onwards and increased in numbers (up to $4.5 \log 10 \mathrm{CFU} / \mathrm{mL}$ at D4) to remain persistent during the remaining time of the experiment. A. pyogenes was identified as previously described[13] and the occurrence was observed on the selective media containing $\leq 0.25 \mu \mathrm{g} /$ $\mathrm{mL}$ enrofloxacin. The latter is in agreement with the study of Yoshimura et al. [14] who found a MIC of $0.5 \mu \mathrm{g} / \mathrm{mL}$ for A. pyogenes and in accordance with a report by Narayanan et al. [15], that support our finding that bovine A. pyogenes strains are able to grow on the selective media containing $16 \mu \mathrm{g} / \mathrm{mL}$ bacitracin (equals approximately 1 $\mathrm{U} / \mathrm{mL}$ ). A. pyogenes is an opportunistic bovine pathogen associated with chronic manifestations of bovine respiratory disease.

In conclusion, a temporary eradication effect of enrofloxacin for $P$. multocida in the nasopharynx of treated calves was present. This is in line with the current PK/PD approach to prevent the selection of resistance, since the AUIC and the $\mathrm{C}_{\max } / \mathrm{MIC}$ ratio measured in the present study largely exceeded the generally accepted thresholds of 125 and 10, respectively. Although confirmation is needed, our results suggest that other respiratory pathogens like A. pyogenes, which are intrinsically less susceptible for enrofloxacin, are able to colonise the upper respiratory tract during fluoroquinolone therapy.

\section{Competing interests}

The authors declare that they have no competing interests.

\section{Authors' contributions}

$\mathrm{BC}$ conceived the study and drafted the manuscript. PD, $\mathrm{GO}, \mathrm{AD}$, and FH participated in the design and coordination of the study. BC, AD and FH performed the microbiological analysis, except for the PFGE and confirmatory identification test, which were done by CK and SS. SC carried out the HPLC, pharmacokinetic/pharmacodynamic analysis, and substantially helped to draft these sections in the manuscript. BC performed and interpreted the statistical analysis. All authors read and approved the manuscript.

\section{Acknowledgements}

We thank Marc Geldhof, Els Defré, Vera Nöding, and Pascal Wassink for excellent technical assistance.

\section{References}

I. Catry B: Pasteurella and Mannheimia species from calves: differentiation and antimicrobial resistance. In $P h D$ thesis Ghent University, Faculty of Veterinary Medicine; 2005.

2. Brunner E, Langer F: Non-parametric variance analysis of longitudinal data München : R Oldenbourg; 1999.

3. Sarasola P, Lees P, AliAbadi FS, McKellar QA, Donachie W, Marr KA, et al.: Pharmacokinetic and pharmacodynamic profiles of danofloxacin administered by two dosing regimens in calves infected with Mannheimia (Pasteurella) haemolytica. Antimicrob Agents Chemother 2002, 46:3013-3019.
4. McKellar QA, Sanchez Bruni SF, Jones DG: Pharmacokinetic/ pharmacodynamic relationships of antimicrobial drugs used in veterinary medicine. J Vet Pharmacol Ther 2004, 27:503-514.

5. Manceau J, Gicquel M, Laurentie M, Sanders P: Simultaneous determination of enrofloxacin and ciprofloxacin in animal biological fluids by high-performance liquid chromatography. Application in pharmacokinetic studies in pig and rabbit. J Chromatogr B Biomed Sci Appl 1999, 726: I75- 184.

6. Sharma PK, Ahmad AH, Sharma LD, Varma R: Pharmacokinetics of enrofloxacin and the rate of formation of its metabolite ciprofloxacin following intravenous and intramuscular single dose administration to male buffalo calves. Vet J 2003, 166:101-104.

7. Kaartinen L, Pyorala S, Moilanen M, Raisanen S: Pharmacokinetics of enrofloxacin in newborn and one-week-old calves. J Vet Pharmacol Ther 1997, 20:479-482.

8. Davis JL, Foster DM, Papich MG: Pharmacokinetics and tissue distribution of enrofloxacin and its active metabolite ciprofloxacin in calves. J Vet Pharmacol Ther 2007, 30:564-57I.

9. McKellar Q, Gibson I, Monteiro A, Bregante M: Pharmacokinetics of enrofloxacin and danofloxacin in plasma, inflammatory exudate, and bronchial secretions of calves following subcutaneous administration. Antimicrob Agents Chemother 1999, 43: $1988-1992$.

10. Schrickx JA, Fink-Gremmels J: Danofloxacin-mesylate is a substrate for ATP-dependent efflux transporters. $\mathrm{Br} J$ Pharmacol 2007, 150:463-469.

II. Schrickx JA, Fink-Gremmels J: Implications of ABC transporters on the disposition of typical veterinary medicinal products. Eur J Pharmacol 2008, 585:510-519.

12. Ismail M, El Kattan YA: Comparative pharmacokinetics of marbofloxacin in healthy and Mannheimia haemolytica infected calves. Res Vet Sci 2007, 82:398-404.

I3. Morrison JR, Tillotson GS: Identification of Actinomyces (Corynebacterium) pyogenes with the API 20 Strep system. J Clin Microbiol 1988, 26: 1865-1866.

14. Yoshimura $\mathrm{H}, \mathrm{Kojima} A$, Ishimaru M: Antimicrobial susceptibility of Arcanobacterium pyogenes isolated from cattle and pigs. J Vet Med B Infect Dis Vet Public Health 2000, 47:139-I 43.

15. Narayanan S, Nagaraja TG, Staats J, Chengappa MM, Oberst RD: Biochemical and biological characterizations and ribotyping of Actinomyces pyogenes and Actinomyces pyogenes-like organisms from liver abscesses in cattle. Vet Microbiol 1998, 61:289-303.

Publish with Bio Med Central and every scientist can read your work free of charge

"BioMed Central will be the most significant development for disseminating the results of biomedical research in our lifetime. "

Sir Paul Nurse, Cancer Research UK

Your research papers will be:

- available free of charge to the entire biomedical community

- peer reviewed and published immediately upon acceptance

- cited in PubMed and archived on PubMed Central

- yours - you keep the copyright 